\title{
Reibschlüssige Greifsysteme für die automatische Handhabung im Materialfluss
}

\author{
DIPL.-ING. ANDREAS WOHLFAHRT \\ FRAUNHOFER-INSTITUT FÜR MATERIALFLUSS UND LOGISTIK IML, DORTMUND
}

Die personalintensive manuelle Durchführung notwendiger Handhabungsaufgaben im Materialfluss ist bedingt durch die oftmals vielfältig auftretenden charakteristischen Eigenschaften der zu behandelnden Stückgutobjekte. Typische Beispiele hierfür sind die Tätigkeiten des Kommissionierens und Sortierens, geprägt durch einen hohen Anteil manueller Tätigkeiten zur Handhabung wie: Vereinzeln, Greifen, Bewegen, Auf- und Abstapeln, Ordnen, Beladen und ähnliche.

Innovative Greifersysteme zur Stückguthandhabung eröffnen neue Möglichkeiten zur weiteren Automatisierung auch in diesem Bereich. Ein Lösungsansatz zur Entwicklung neuer Greifsysteme ist es, gezielt Friktionskräfte in Oberflächenbereiche des zu greifenden Objektes einzubringen und diese für den Handhabungsvorgang z.B. als Haltekräfte zu nutzen. Bei guten Reibwerten und entsprechender Anordnung der Wirkelemente können Objekte auch dann rein reibschlüssig gegriffen werden, wenn deren Oberfläche nur seitlich und von oben zugänglich ist, wie etwa Kartons im Packmusterverbund auf der Palette.

The widely differing characteristics of piece goods often require a manual and labour-intensive handling within the material flow. Typical activities are order-picking and sorting with a high share of manual handling like decollating, gripping, moving, stacking, rearranging, loading, etc. Innovative gripper systems for the handling of piece goods offer new potentials for further automation.

One gripper concept includes the direct transmission of friction forces into the surface of the object to be gripped and use of these forces, e.g. as gripping force. When the friction values are good and the elements are arranged correctly objects can be gripped frictionally when they can be approached only from above or from the side, e.g. boxes in a composite packaging pattern on a pallet.

\section{Rationalisierungspotential bei der Stückguthandhabung}

Die Vielzahl oftmals gleichzeitig auftretender Erscheinungsformen von Konsumgütern, die durch ein System bewältigt werden müssen, behindert eine durchgreifende Automatisierung in diesem Bereich der Stückguthandhabung. Die Verschiedenartigkeit der Güter zeigt sich in Größe, Form, Masse, Verpackungsart, Bereitstelltoleranzen, Festigkeit bzw. Empfindlichkeit, Instabilität, Schwerpunktlage und weiteren artikelspezifischen Eigenschaften des zu handhabenden Gutes. 

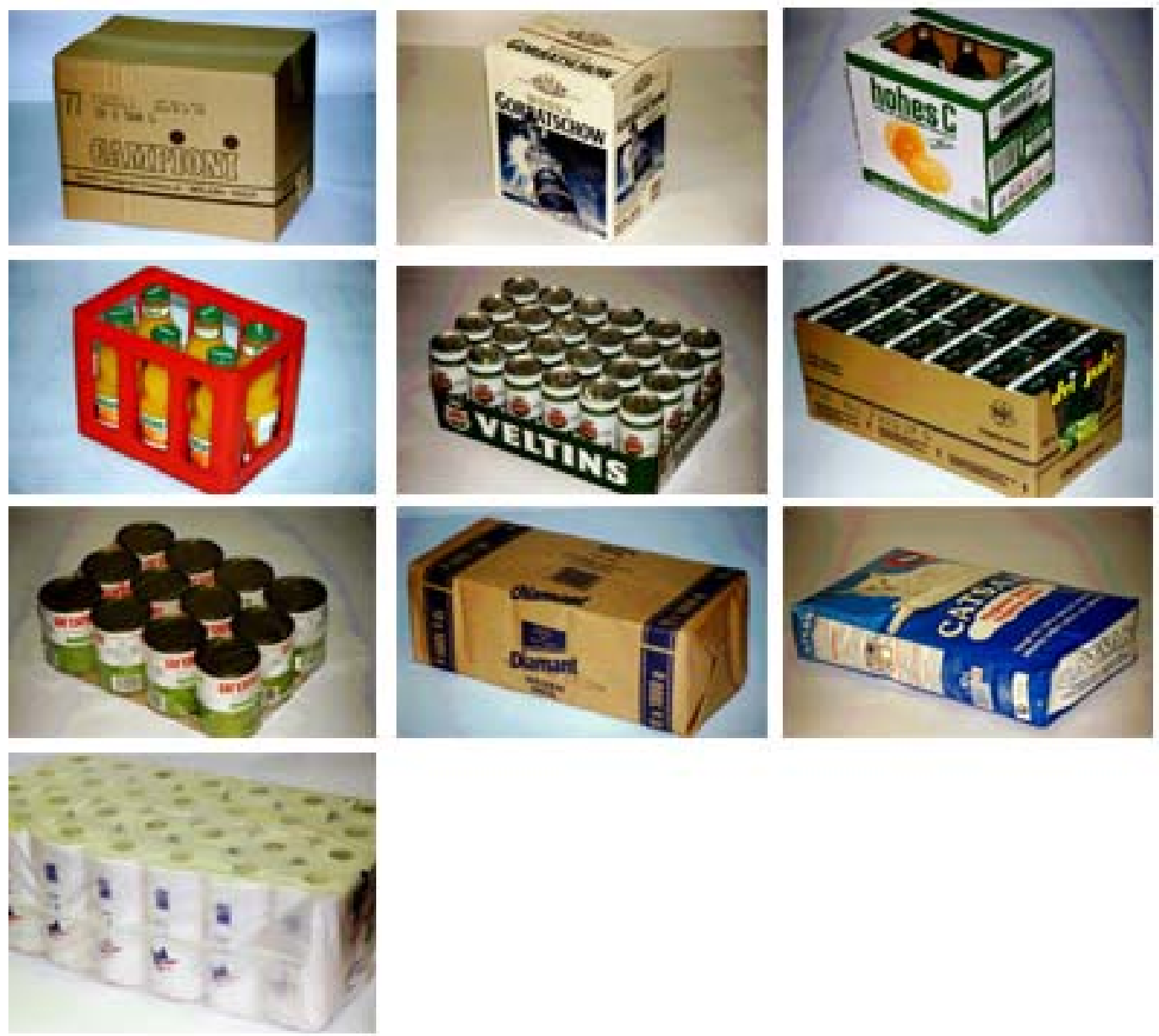

Abb. 1: Typische Erscheinungsformen von Konsumgütern

Die bei manueller Handhabung eingesetzten sensorischen und motorischen Fähigkeiten des Menschen sind bei der Automatisierung nur mit hohem Aufwand realisierbar. Schlüsselkomponenten zur Nutzung dieser Rationalisierungspotentiale sind intelligente Greifsysteme, die spezifische Objekteigenschaften bei der Handhabung berücksichtigen.

\section{Vereinzelung von Stückgütern aus dem Verbund}

Besondere Problemstellung ist das Vereinzeln im Verbund bereitgestellter Güter (Abb. 2). Beispiel hierfür ist das Entnehmen gestapelter Artikel von Paletten. Für Einzelzugriffe stehen nicht immer gegenüberliegende Greifflächen zur Verfügung, wie in Abb. 3 erkennbar. Zum Ergreifen des rot gekennzeichneten Artikels sind nur drei Greifflächen zugänglich, die gegenüberliegenden Flächen sind verdeckt. Hierdurch wird der Einzelzugriff auf den Artikel erschwert. 


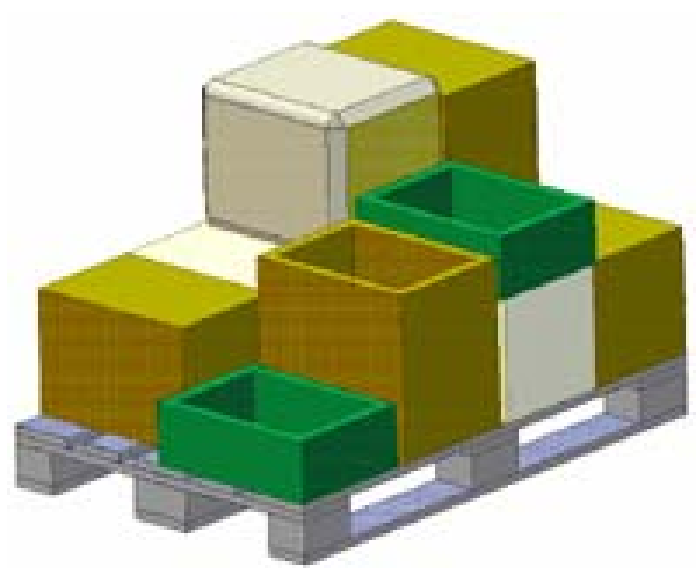

Abb. 2 : Im Verbund gestapelte Objekte

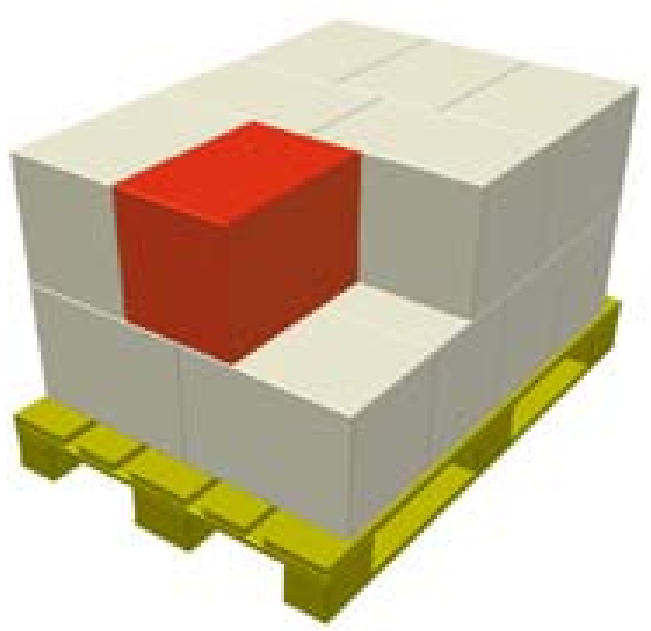

Abb. 3: Nur drei Wirkflächen zum Greifen zugänglich

Auch beim Zugriff auf ganze Lagen eines Stapels können für das Greifsystem besondere Anforderungen auftreten. Verzahnung der Stapel, hervorgerufen durch Packmustertoleranzen, dreidimensional verschachtelte Packmuster, Packmuster mit Kaminbildung, zur Ladungssicherung verklebte Objekte und andere sind die Ursache hierfür. Ein Abschieben ganzer Lagen ist nur in besonderen Fällen möglich.

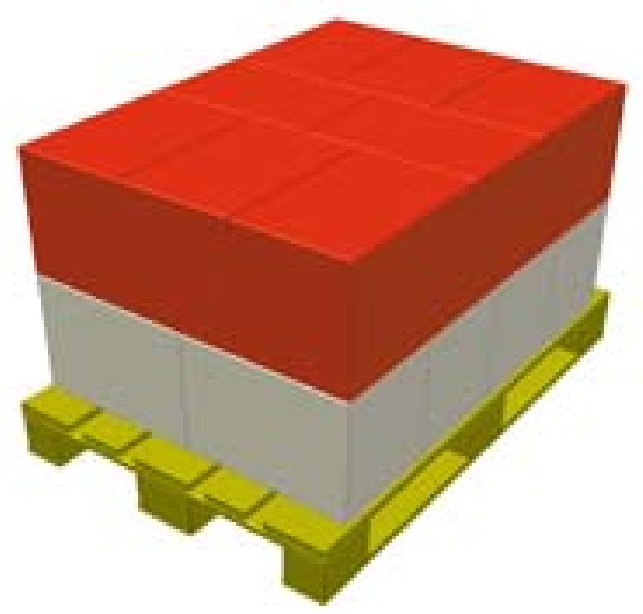

Abb. 4: Lagenweises Entstapeln 


\section{Stand der Technik}

Vielfältig zur Anwendung kommen Saug- und Klemmgreifer. Viele Handhabungsaufgaben können mit diesen Greifsystemen jedoch nicht oder nur unbefriedigend gelöst werden. Dies gilt insbesondere für nicht saugbare, im Verbund liegende (d.h. dicht nebeneinander liegende) Objekte.

\section{Sauggreifer}

Sehr verbreitet sind Vakuumgreifer, insbesondere für Palettierung und Depalettierung. Durch unterschiedliche Ausgestaltung und intelligente Ansteuerung finden sie ein weites Anwendungsspektrum.

Sauggreifer sind für den Einzel- und Lagenzugriff geeignet. Der Zugriff auf Verbundstapel ist möglich, da im Idealfall nur eine Fläche zugänglich sein muss. Sie sind in vielen Varianten und für viele Aufgabenstellungen verfügbar. Für extreme Einsatzfälle sind Anwendungen mit über 100 Einzelsaugern bekannt (Abb. 5). Sauggreifer sind jedoch nur für Greifgut mit hinreichend steifen, ansaugbaren Oberflächen geeignet.

In der Regel ist ein hoher Energieverbrauch zur Aufrechterhaltung des Unterdruckes erforderlich. Großflächig wirkende Lagensauger mit hohen Saugvolumenströmen kommen zur Anwendung. Zur Kompensation der Druckverluste durch die auftretenden Leckvolumenströme werden oftmals Gebläse mit hoher Leistung benötigt. Beispiele mit 6kW Saugleistung und mehr sind bekannt. Hoher Energieverbrauch, Schallemission, Luftverwirbelungen und Staubentwicklung sind die Nachteile des Sauggreifens.

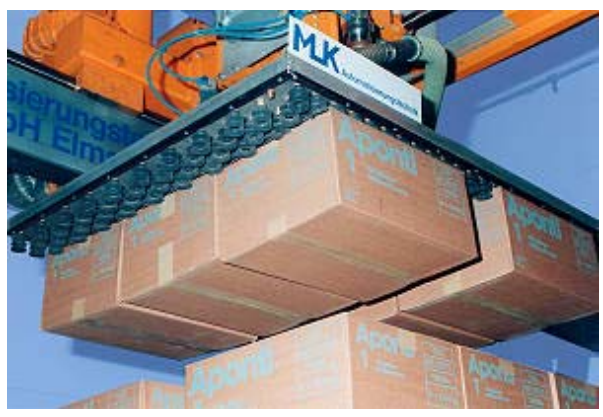

Abb. 5: Sauggreifer, hier Anwendung als Lagengreifer mit einer Vielzahl an Einzelsaugelementen [Quelle: http://www.muk-elmshorn.de - Stand 04/2005]

\section{Klemmgreifer}

Klemmgreifer sind als klassisches Greifsystem in vielen Erscheinungsformen verfügbar. Vielfältig im Einsatz sind Zwei- oder Mehrfinger- bzw. -backengreifer, die das Gut formschlüssig oder durch Klemmung für die anstehende Handhabungsaufgabe fixieren. Häufig angewendete Ausführungsformen sind Kniehebelgreifer, Parallelgreifer und Zangengreifer.

Klemmgreifer sind jedoch nicht zum Vereinzeln im Verbund liegender Güter geeignet, da sie frei zugängliche, gegenüberliegende Greifflächen des Gutes benötigen. Klemmgreifer wirken reibschlüssig und oder formschlüssig bei entsprechender Formgebung der Greifbacken.

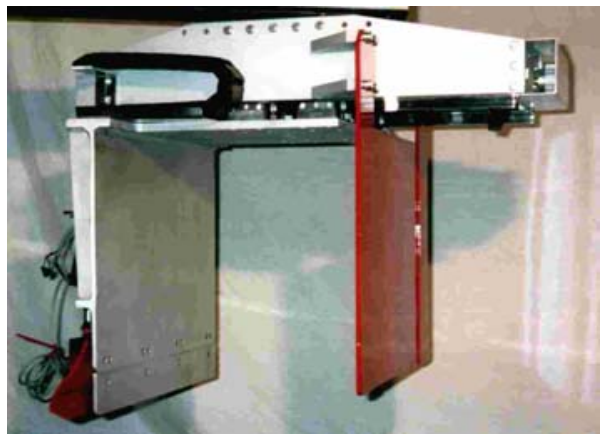


Abb. 6: Parallelklemmbackengreifer [Quelle: http://www.holmatec.de - Stand: 04/2005]

\section{Sondergreifer}

Sondergreifer für spezielle Anwendungen sind in der Regel Spezialkonstruktionen für eingeschränkte Artikelspektren. Sie arbeiten meist formschlüssig und sind nicht universell einsetzbar.

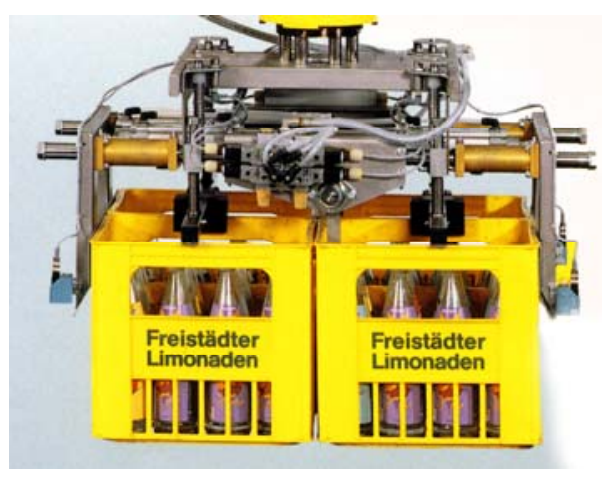

Abb. 7: Spezialgreifer für Getränkekästen [Quelle: KRONES AG, Neutraubling]

\section{Aufwälzgreifer}

Seit einigen Jahren wird der Lösungsansatz des Aufwälzgreifens verfolgt. Sie sind speziell für Depalettier- und Kommissioniervorgänge entwickelt worden, erlauben den Einzelzugriff auf Verbundstapel und sind einsetzbar für unterschiedlichste Güter.

Auch bei diesem Greifsystem spielen Reibungskräfte eine wichtige Rolle. Das wichtigste Element des Aufwälzgreifers ist das angetriebene Friktionselement ausführbar als Rolle oder Riemen. Es ermöglicht das Aufsteigen des Objektes auf den Greifer. Hierzu wird eine Friktionsrolle oder ein Friktionsriemen stirnseitig an das zu greifende Objekt angedrückt, das Objekt steigt an der Greifrolle hoch, ein Unterfahren des Objektes durch den Greiferschlitten wird bewirkt.

Ist die Umfangsgeschwindigkeit der Riemen beim Unterfahren des Objektes gleich der Zustellgeschwindigkeit des Greiferschlittens, wird ein schlupfloses Unterfahren des zu greifenden Objektes ermöglicht. Die Bezeichnung Aufwälzgreifer basiert auf diesem Verhalten des Greifsystems.

Ein elementares Aufwälzgreifsystem zur Handhabung von Papierriesen ist in Abbildung 8 dargestellt. Das Greifsystem arbeitet mit gummibeschichteten Riemen als Friktionselement. Das im Bild sichtbare Messsystem zur Erfassung der Stapelhöhe ermöglicht eine optimale Ausrichtung zwischen Greifer und aufzunehmenden Objekt in der Vertikalen.

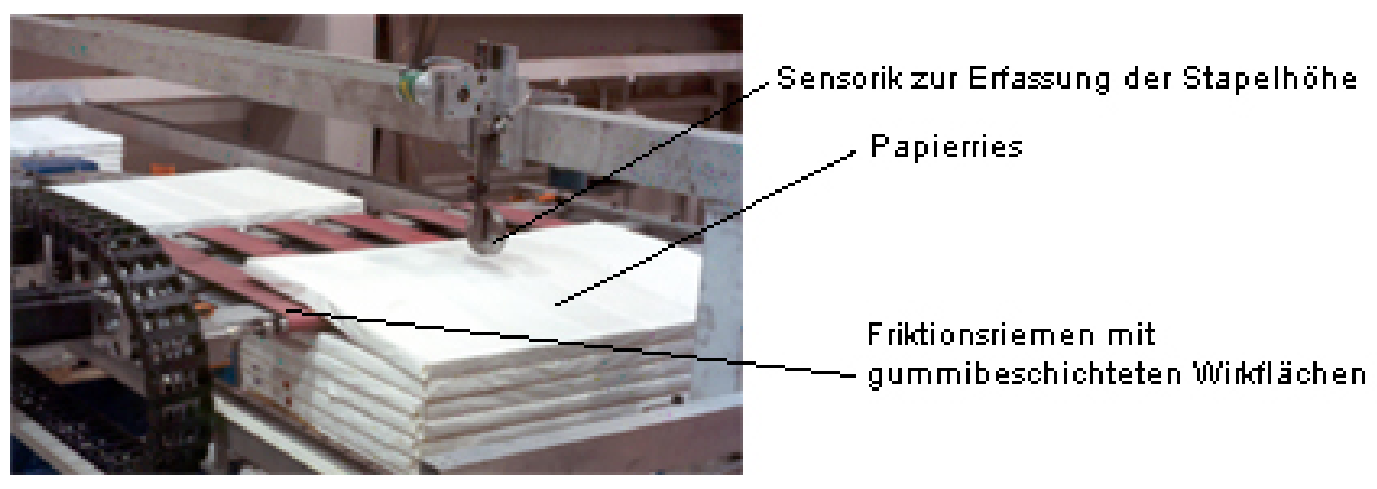

Abb. 8: Elementarer Aufwälzgreifer für Papierriese 
Durch den Einsatz weiterer zusätzlicher Hilfseinrichtungen und Anwendung artikelspezifischer Greifstrategien zur Unterstützung des Greifprozesses kann das Greifsystem universell für sehr verschiedenartige Objekte eingesetzt werden.

Beispielsweise wird der in Abbildung 10 dargestellte Gegenhalter verwendet, um ein Verrutschen der Artikel beim Greifen zu verhindern. Ebenso kann durch entsprechende Zustellbewegungen des Gegenhalters ein Ankippen des zu greifenden Objektes bewirkt werden. Durch diese Strategie wird das Aufnehmen und Unterfahren des Objektes begünstigt. Abbildung 9 zeigt das Foto eines Aufwälzgreifers mit Gegenhaltereinsatz.

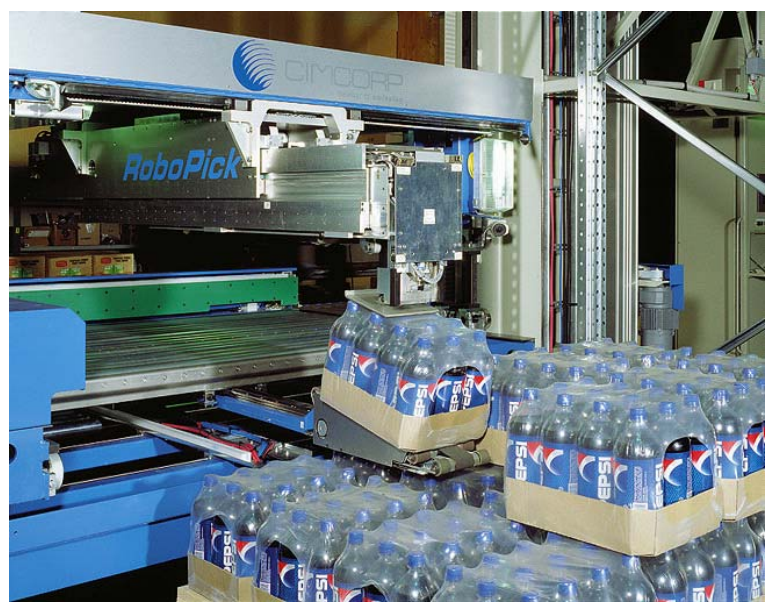

Abb. 9: Aufwälzgreifer zur Kommissionierung von Verbrauchsgütern [Quelle: Fa. Swisslog Cimcorp, Ulvila, Finnland]

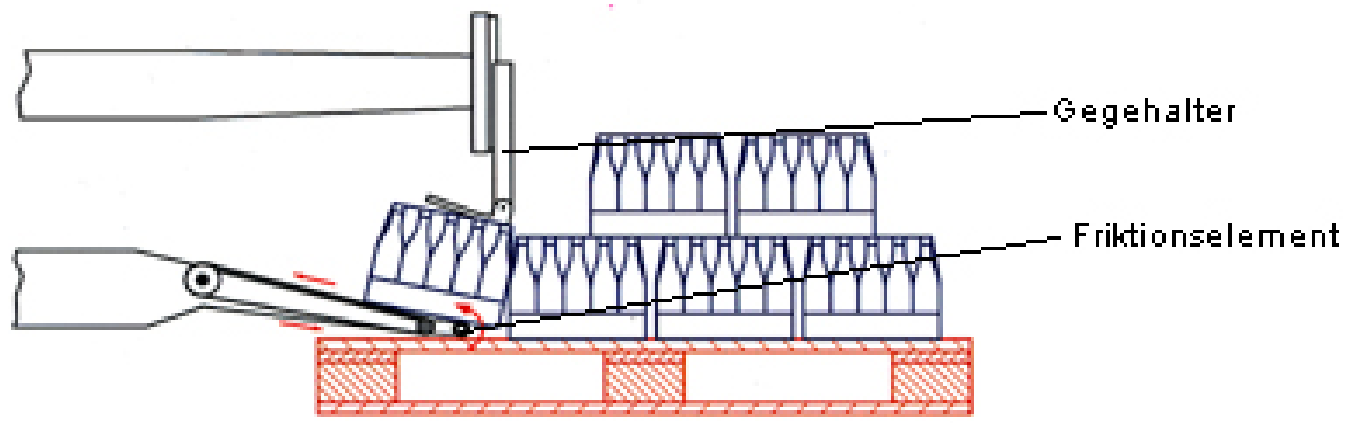

Abb. 10: Prinzipbild des Aufwälzgreifers für Verbrauchsgut

\section{Reibschlüssig wirkender Riemenklemmgreifer}

Ein ausschließlich kraftschlüssig durch Reibung wirkendes Greifsystem ist der neuartige, im Folgenden vorgestellte Riemenklemmgreifer. Das entsprechend seiner Wirkungsweise als Traction-Gripper bezeichnete Greifsystem ist gekennzeichnet durch Reibriemen, die beispielsweise in einem Winkel von $90^{\circ}$ zueinander angeordnet sind (Abb. 11 und 12). 


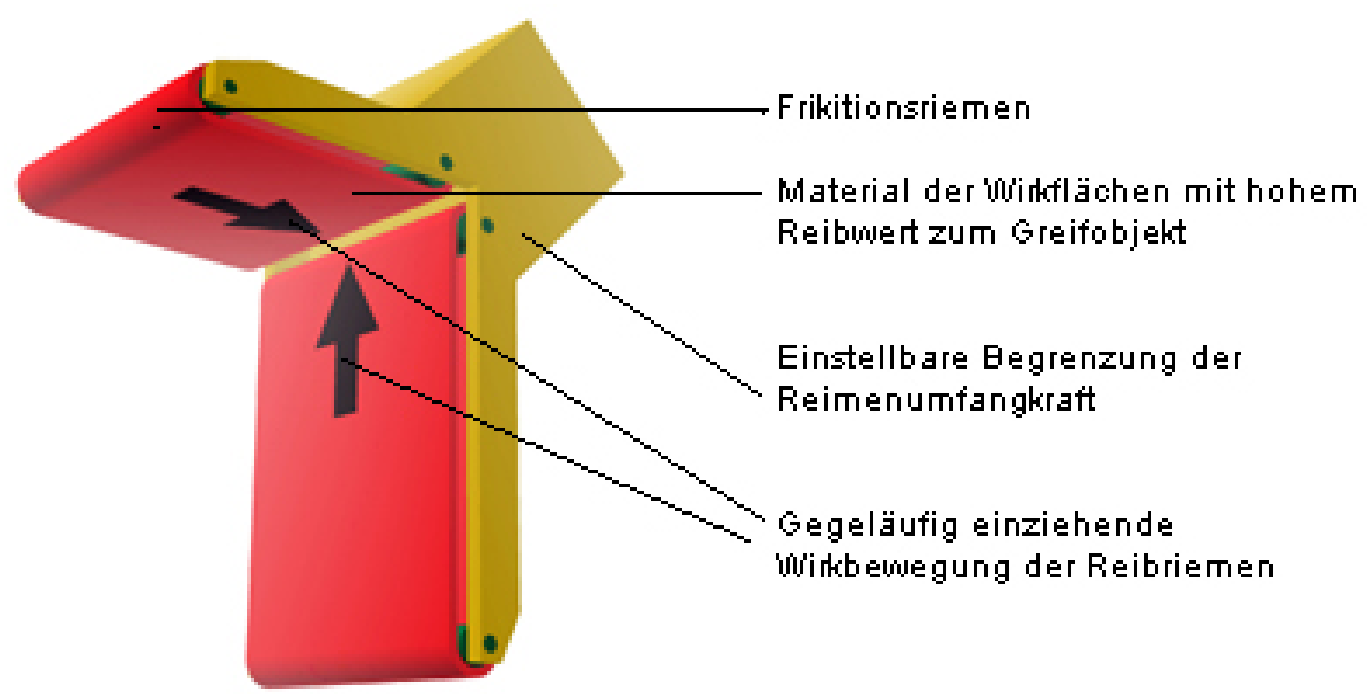

Abb. 11: Wirkprinzip des Traction-Grippers

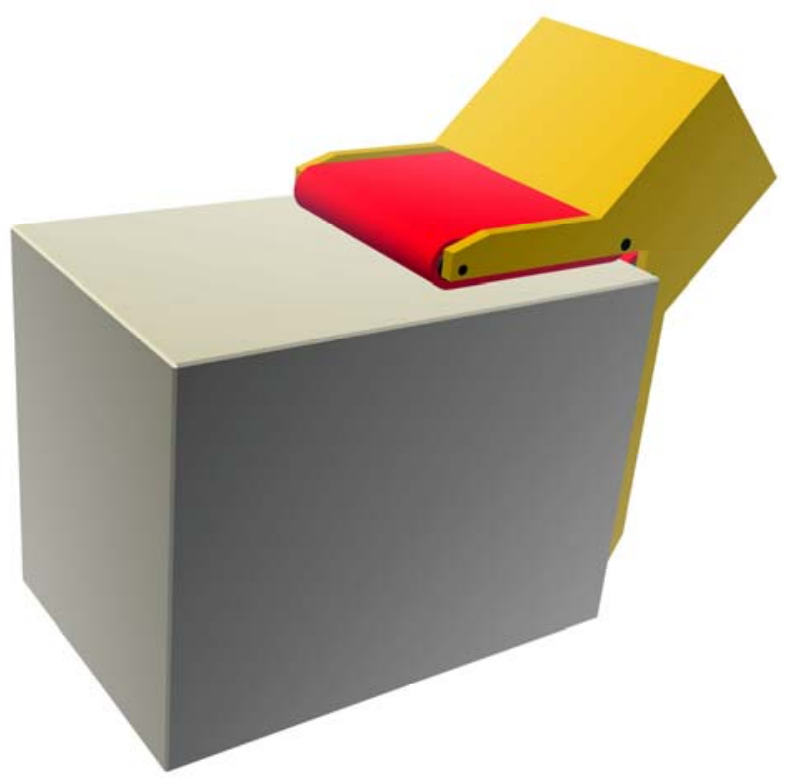

Abb. 12: Prinzipbild Traction-Gripper mit quaderförmigem Objekt

Die Gutaufnahme erfolgt reibschlüssig nach Berührung der Wirkflächen des Greifers mit der Objektoberfläche durch die gegenläufig einziehende Bewegung der Reibriemen. Ein hoher Reibwert zwischen Greifobjekt und Reibriemen ist Voraussetzung für die Funktion des Greifsystems.

Setz- oder Schlupferscheinungen, die zu einem Verlust des Reibschlusses führen könnten, werden durch die ständig nachstellenden Reibriemen kompensiert. Ein Überschreiten der zulässigen Umfangskraft wird durch die einstellbare Kraftbegrenzung vermieden.

\section{Prototyp}

Zur Verifikation des Prinzips wurde am Fraunhofer IML ein Prototyp eines Traction-Grippers aufgebaut (Abb. 13). Der Greifer ist mit jeweils sechs horizontal und sechs vertikal angeordneten Wirkriemen ausgerüstet. Die Zugkraftbegrenzungen der Einzelriemen wirken unabhängig voneinander. Riemenumfangsgeschwindigkeiten und Zugkraftbegrenzungen sind artikelspezifisch 
einstellbar. Als Werkstoff zur Beschichtung der Wirkflächen der Reibriemen kommt ein Naturkautschukprodukt mit hohem Reibwert neben vielen anderen Materialien zur Anwendung.
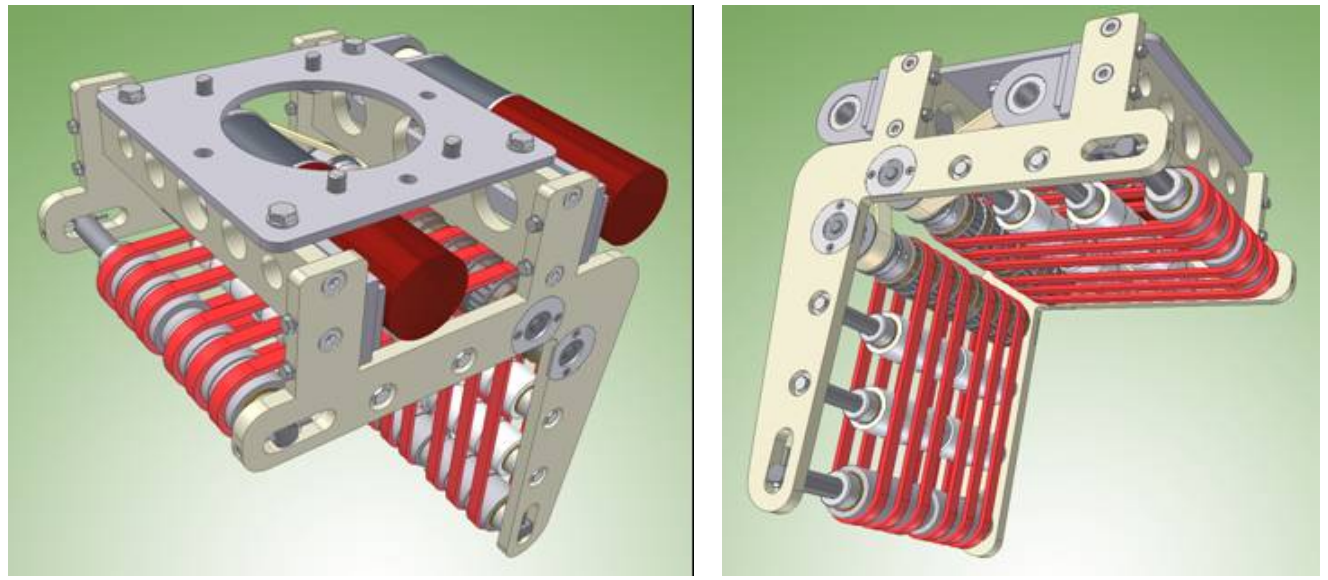

Abb. 13: CAD-Modell des Prototyp

\section{Konstruktive Gestaltung des Prototypgreifers}

Der Prototyp des Greifsystems ist einfach aufgebaut und kostengünstig herstellbar. In Abbildung 14 ist die Gestaltung der Riemenkraftbegrenzungen dargestellt. Zur Übertragung der Drehbewegung der Keilwelle auf die Mitnehmerscheiben sind diese mit der Keilwelle permanent im Eingriff.

Die zwischen den Mitnehmerscheiben angeordneten nadelgelagerten Riemenscheiben besitzen keine formschlüssige Drehverbindung zur Keilwelle. Die so verdrehbar auf der Keilwelle angeordneten Riemenscheiben werden reibschlüssig durch die Mitnehmerscheiben angetrieben. Mittels Nutmutter und Tellerfedern werden die Riemenscheiben mit den Mitnehmerscheiben axial verspannt. Das maximale Rutschmoment der auf diese Weise gebildeten Rutschkupplung wird durch die Höhe der Verspannung bestimmt und damit die maximale Umfangskraft in den Riemen.

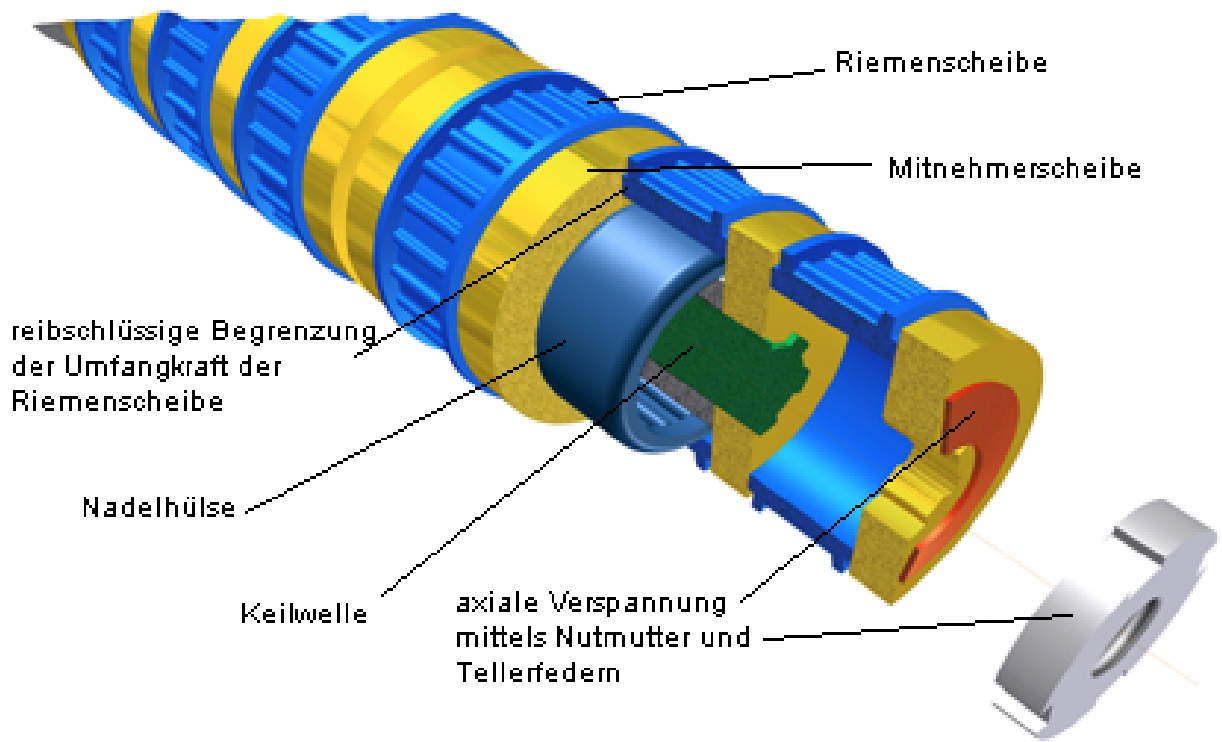

Abb. 14: Rutschkupplung zur Begrenzung der Riemenumfangskraft

\section{Eigenschaften des neuen Greifsystems}

- Die Handhabung verschiedenartiger Objekte ist möglich. Beispiele sind oben offene Artikel oder solche mit unebener Oberfläche. Auch luftdurchlässige, nicht ansaugbare Objekte können mit dem Greifsystem ergriffen werden. 
- Die Verfügbarkeit gegenüberliegender Greifflächen ist nicht erforderlich. das Greifsystem wurde speziell für den Einzelzugriff aus dem Verbund konzipiert.

- Der Energieverbrauch durch langsam drehende Reibriemen ist sehr gering, die Leistungsaufnahme der Motoren des Prototypen liegt unter $50 \mathrm{~W}$.

- Eine artikelspezifische Anpassung des Greifsystems für spezielle Aufgabenstellungen kann durch Einstellung der maximalen Riemenumfangskräfte und der Riemengeschwindigkeit erfolgen.

- Eine großflächige Krafteinleitung wird durch die Vielzahl der parallel zueinander laufenden Greifriemen bewirkt, eine gleichmäßige Krafteinleitung durch die unabhängig voneinander ansprechenden Kraftbegrenzungen. Auf diese Weise ist ein artikelschonendes Handling der Greifobjekte realisierbar.

- Der einfache Aufbau des Systems ermöglicht kostengünstige Greiferlösungen.

- Das Prinzip kann als Grundlage für andere oder weiterführende Entwicklungen genutzt werden.

\section{Theorie zur Ermittlung der Riemenkräfte}

Für ausgewählte Fälle wird im Folgenden die rechnerische Bestimmung der erforderlichen Antriebskräfte in Abhängigkeit des Reibwertes durchgeführt. Alle Riemenkraftangaben sind auf das Eigengewicht des Greifobjektes normiert.

\section{Fall 1: Homogener Würfel hängt frei im Riemenklemmgreifer}

Ermittlung der zum Halten erforderlichen Riemenumfangskräfte F in Abhängigkeit von Reibwert $\mu$ und Gewicht $\mathrm{G}$.

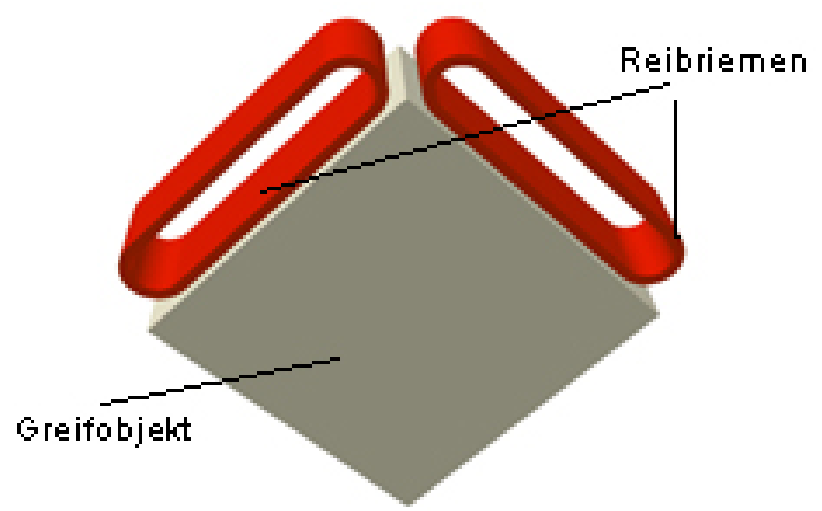

Abb. 15: Belastungsskizze

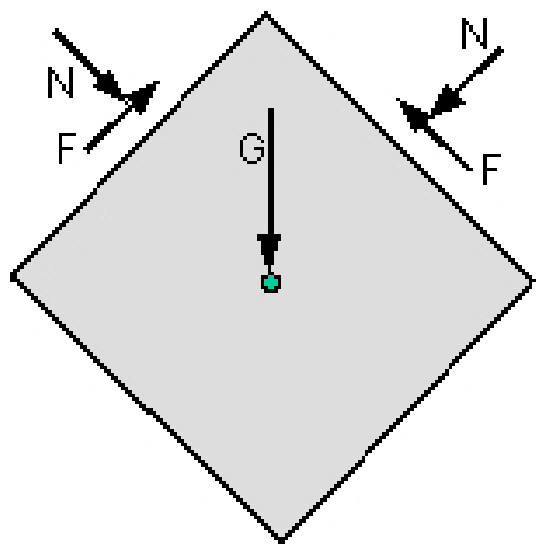

Abb. 16: Freikörperbild 


$$
\text { Auswertung der Gleichgewichtsbedingungen: } \quad F=\frac{\mu}{\mu-1} \cdot \frac{1}{\sqrt{2}} \cdot G
$$

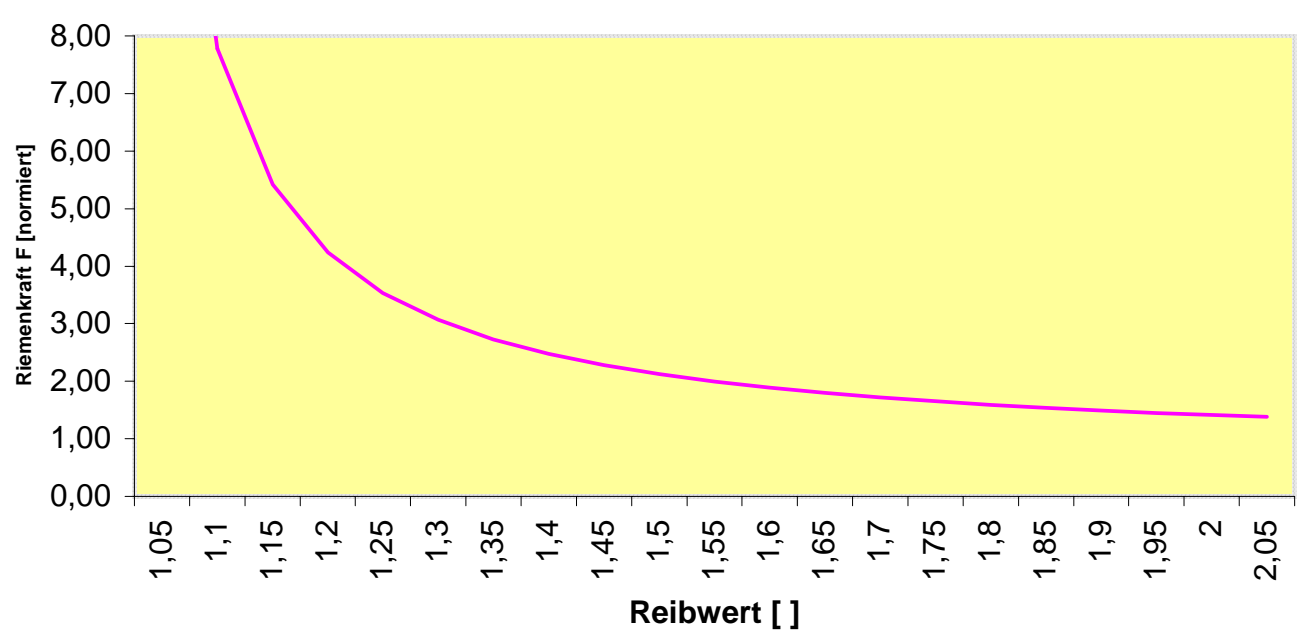

Abb. 17: Funktion $\mathrm{F}(\mu)$

Man erkennt deutlich, wie auch in allen weiteren vorgestellten Fällen eine entscheidende Voraussetzung für das Funktionieren des Greifprinzips:

$$
\mu \succ 1
$$

Der Reibwert zwischen Riemenwirkfläche und Objekt muss größer als 1 sein.

\section{Fall 2: Quaderförmiges Packstück im vertikal ausgerichteten Greifer}

Ermittlung der zum Halten erforderlichen Riemenumfangskräfte $\mathrm{F}$ für homogene Quader bei vertikal ausgerichtetem Greifer in Abhängigkeit von Reibwert $\mu$ und Gewicht G.

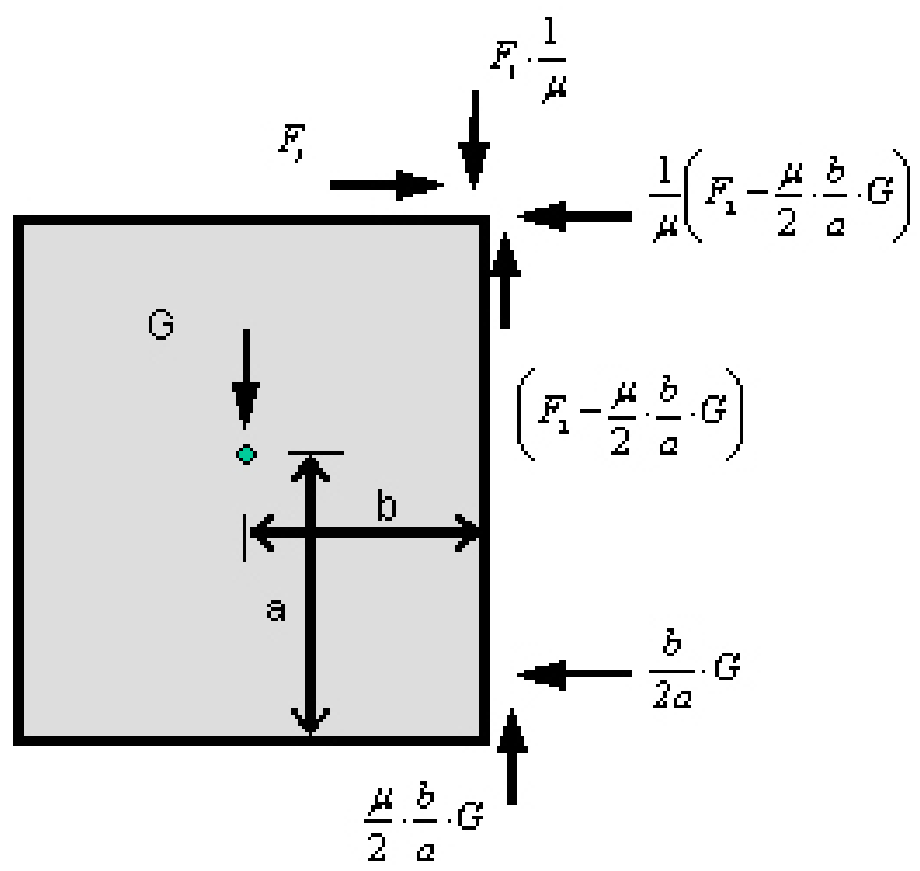

Abb. 18: Freikörpermodell 


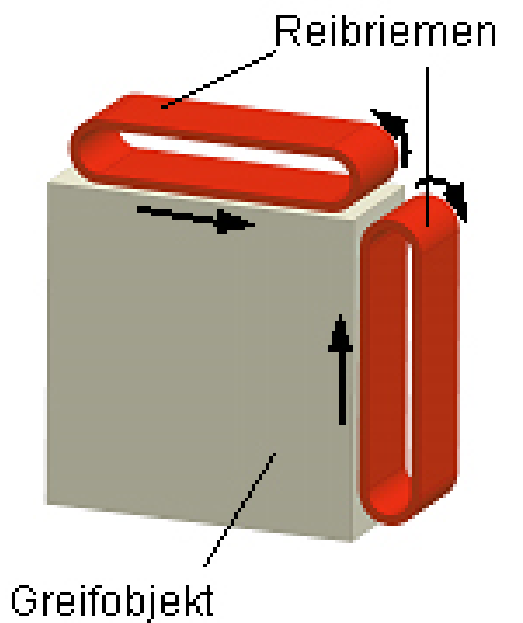

Abb. 19: Belastungsskizze

Auswertung der Gleichgewichtsbedingungen:

Umfangskraft im Horizontalriemen:

$F_{1}=\left(\frac{\mu}{\mu^{2}-1}\right) \cdot G$

Umfangskraft im Vertikalriemen:

$$
F_{2}=\left(\frac{\mu^{2}}{\mu^{2}-1}\right) \cdot G
$$

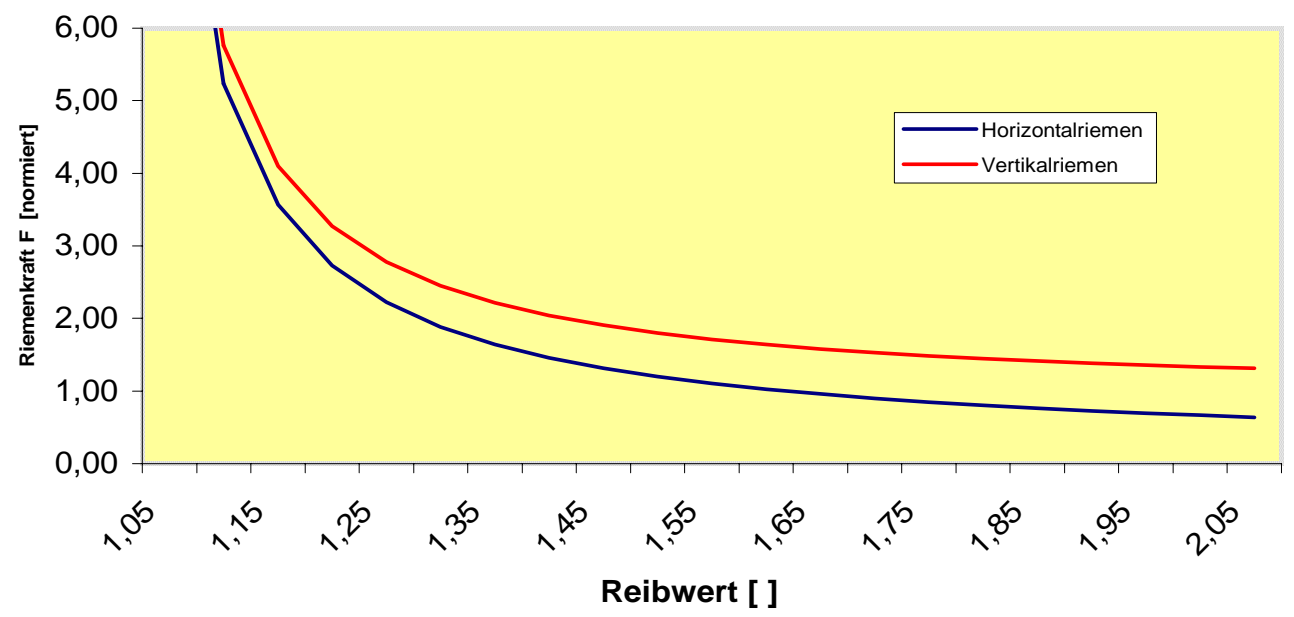

Abb. 20: Funktion $F(\mu)$

Die erforderliche Riemenumfangskraft im Vertikalriemen ist größer als die im Horizontalriemen.

Fall 3: Kugel- und zylinderförmige Packstücke im vertikal ausgerichteten Greifer

Ermittlung der zum Halten erforderlichen Riemenumfangskräfte F für kugel- und zylinderförmige homogene Packstücke bei vertikal ausgerichtetem Greifer in Abhängigkeit von Reibwert $\mu$ und Gewicht $\mathrm{G}$. 


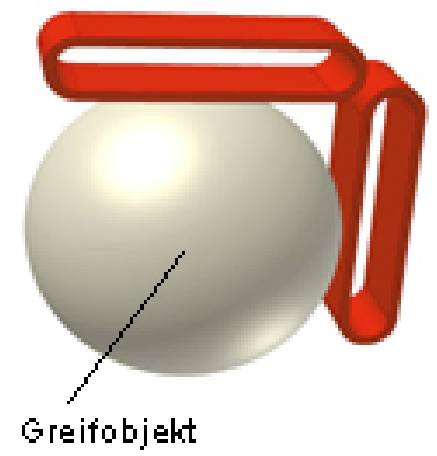

Abb. 21: Belastungsskizze

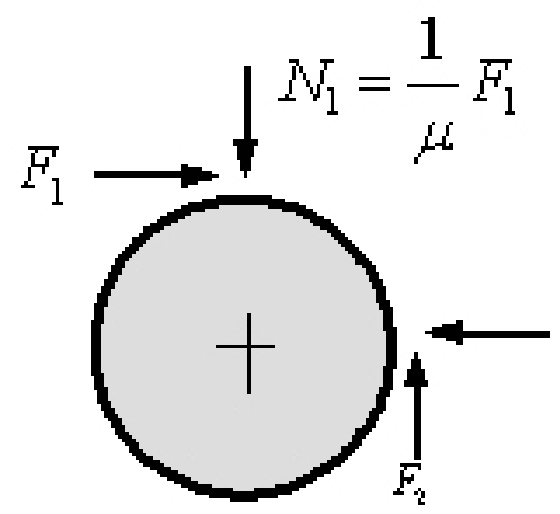

Abb. 22: Freikörperbild

Auswertung der Gleichgewichtsbedingungen:

Umfangskraft im Horizontal- und Vertikalriemen: $\quad F_{1}=F_{2}=\left(\frac{\mu}{\mu-1}\right) \cdot G$

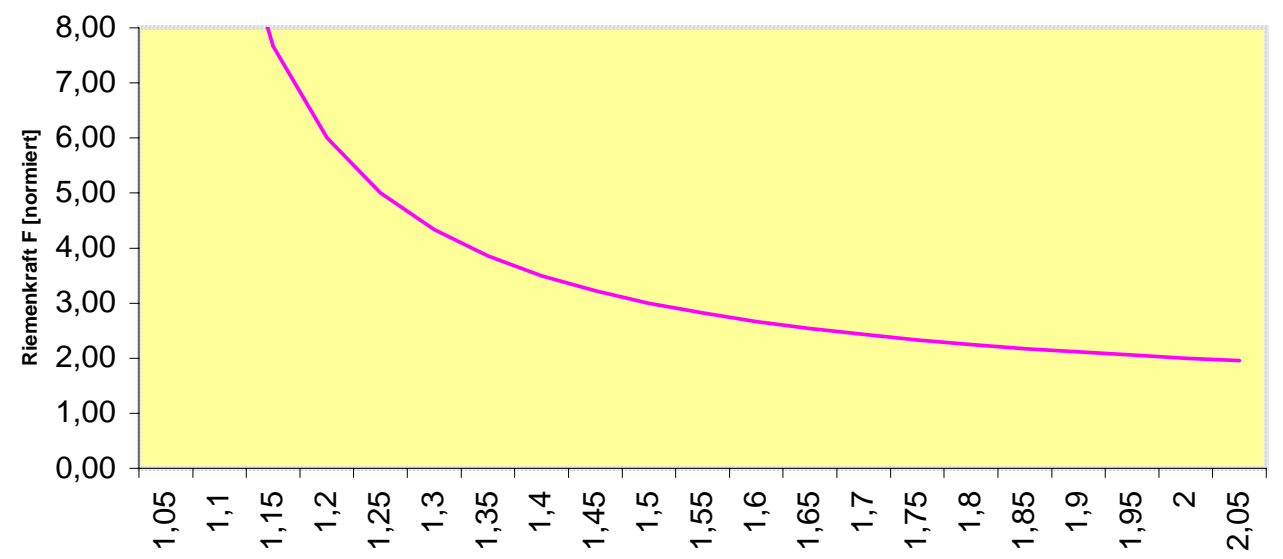

Reibwert [ ]

Abb. 23 Funktion $F(\mu)$

Im Vergleich zu den anderen betrachteten Objekten ergeben sich rechnerisch die höchsten der ermittelten Kräfte. 


\section{Fall 4: Quaderförmiges Packstück im angestellten Greifer}

Ermittlung der zum Halten erforderlichen Riemenumfangskräfte F für homogene Quader, bei angestelltem Greifer und angestelltem Objekt in Abhängigkeit von Reibwert $\mu$ und Gewicht G.

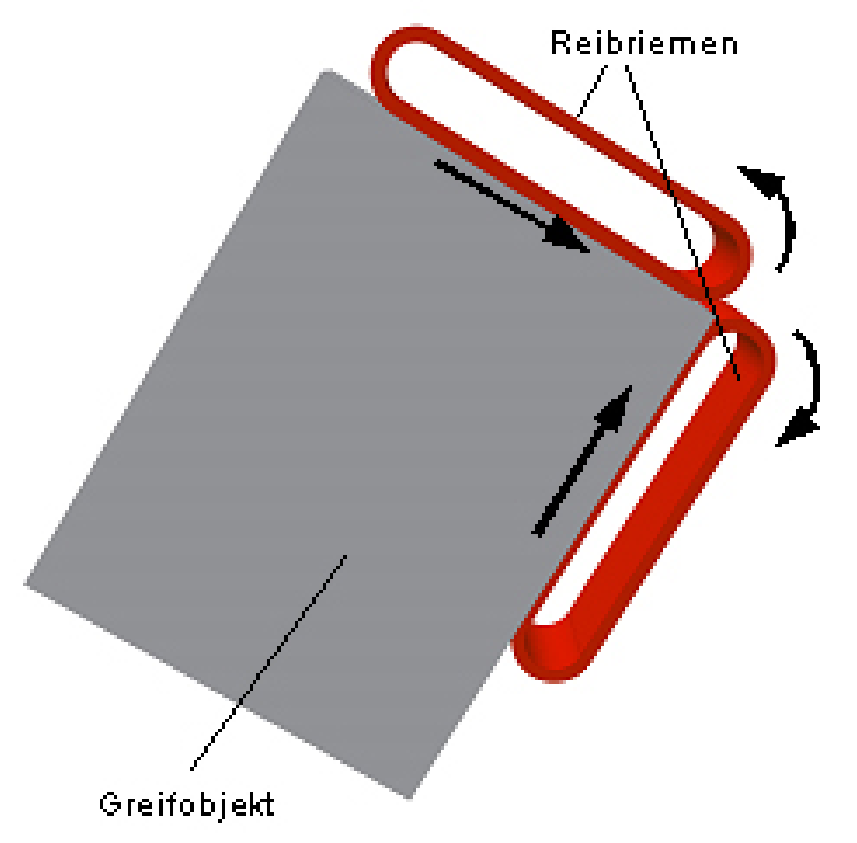

Abb. 24: Belastungsskizze

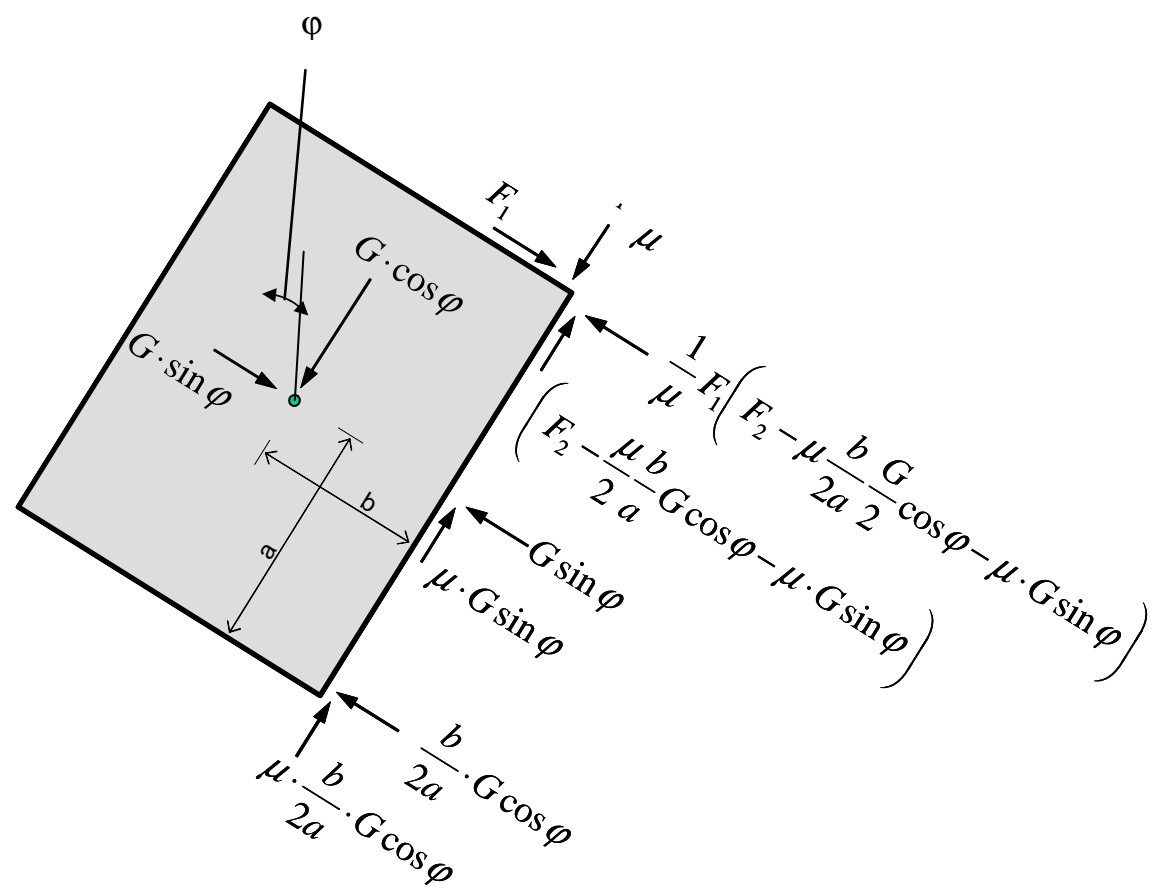

Abb. 25: Freikörperbild 
Auswertung der Gleichgewichtsbedingungen:

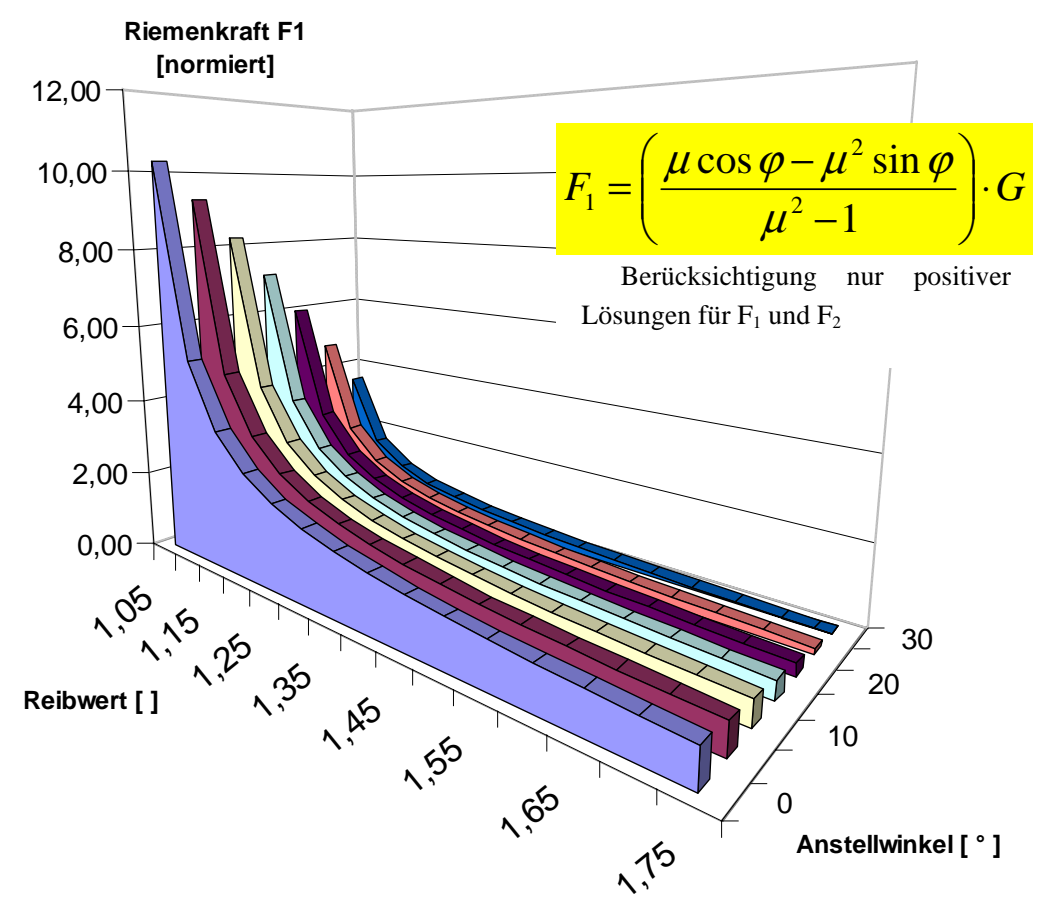

Abb. 26: Umfangkraft im Horizontalriemen

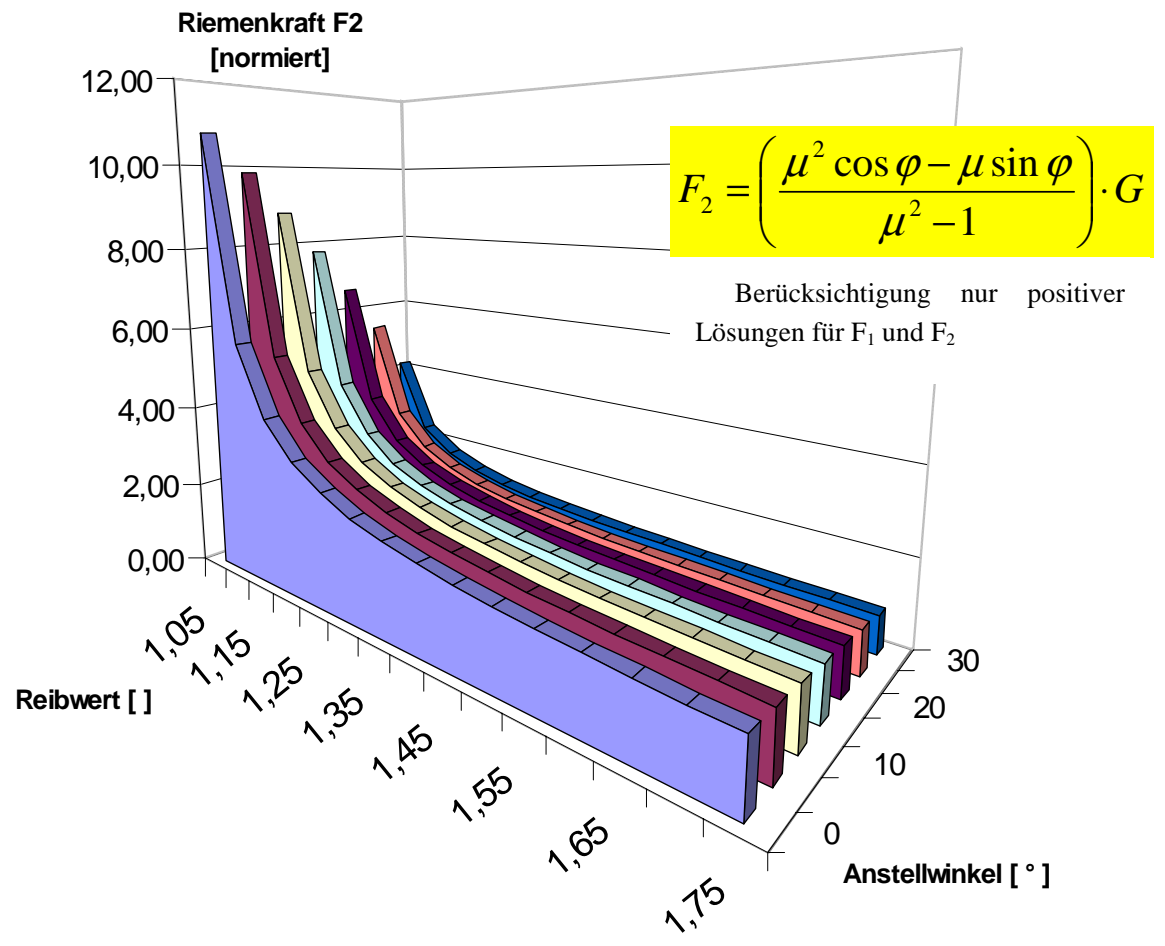

Abb. 27: Umfangkraft im Vertikalriemen 


\section{Strategische Planung des Bewegungs- und Aktionsablaufes}

Eine Berücksichtigung der Greifereigenschaften bei der Planung des Bewegungs- und Aktionsablaufes der Handhabungsoperation ermöglicht eine Optimierumg des Haltevermögens des Greifsystems. Eine Anstellung des Greifers kann beispielsweise bei der Translation die erforderlichen Greifkräfte verringern. Ein entsprechender Bewegungs- und Funktionsablauf ist im Folgenden skizziert:

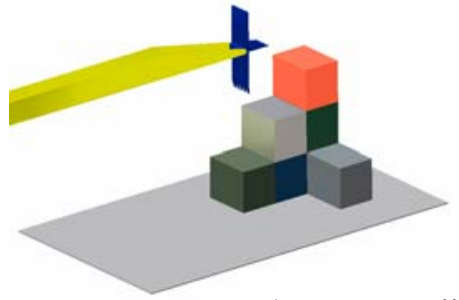

Ausgangsstellung

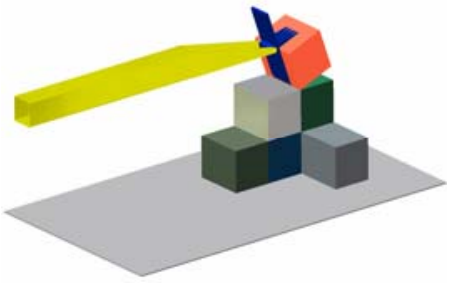

Kippen

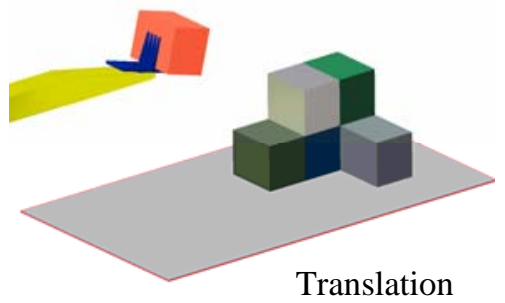

Translation

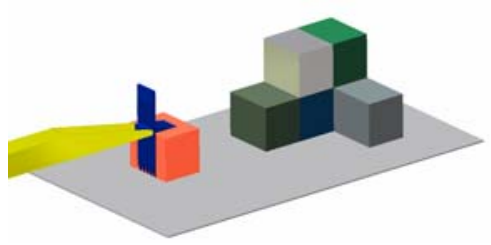

Abkippen

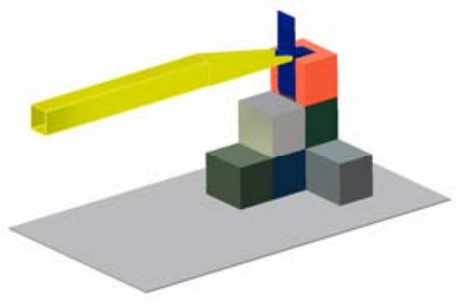

Positionieren

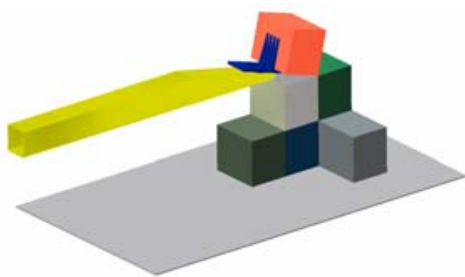

Kippung abschließen

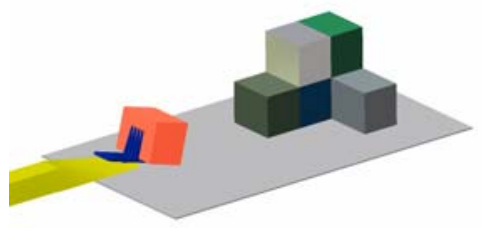

Positionieren

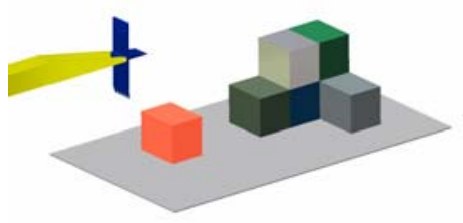

Freifahren

Abb. 28: Bewegungs- und Funktionsablauf

Die Anstellung des Objektes führt durch formschlüssige Unterstützung des Objektes zu stärkerem Halt des Objektes im Greifer. Bei der Translation können so höhere Kräfte aufgenommen werden. 


\section{Gültigkeit der Modellrechnung}

Die vorgestellte Modellrechnung geht von coulombscher Festkörperreibung aus und gilt streng genommen nur für Festkörper. Für die coulombsche Festkörperreibung gelten einfache Zusammenhänge:

$$
F_{R}=\mu \cdot F_{N} \text { und } \mu_{G} \prec \mu_{H}
$$

Bei Verwendung von Gummiwerkstoffen müssen komplexere Gesetzmäßigkeiten berücksichtigt werden, da Gummi kein Festkörper, sondern eher eine sehr viskose Flüssigkeit ist. Adhäsions-, Hysterese und Kohäsionsreibanteile bestimmen den nicht konstanten Reibwert. Dieses Verhalten von Gummi wird in dem Aufsatz von Klüppel und Alshuth [Klüppel98] beschrieben.

Der dominierende Adhäsionsreibungsanteil mit höherem Gleit- als Haftreibwert wird in Versuchen mit dem Traction-Gripper experimentell bestätigt.

Ebenso zeigt sich ein mit der Schlupfgeschwindigkeit zunächst ansteigender Reibwert. Untersuchungen zu adhäsiven Reibeigenschaften von Gummiproben wurden von Eberhardsteiner, Fidi und Liederer durchgeführt [Eberhardsteiner98].

Als Werkstoff für die Wirkflächen der Reibriemen wurde zwecks Erzielung eines hohen Reibwertes „Linatex“, ein Naturkautschukprodukt ausgewählt. Dieser Werkstoff besitzt einen Härtegrad von $40^{\circ}$ shore A, einen hohen Reibwert zu vielen Materialien und ist bewährt bei Transport- und Abzugsbändern für Anwendungen mit hoher Friktion und hoher Beschleunigung.

\section{Stand der Forschung}

Reibschlüssig wirkende Greifer mit nachstellenden Wirkflächen zum Schlupfausgleich sind nicht bekannt. Forschungen zum Reibverhalten von Gummi zu verschiedenartigen Materialien werden insbesondere im Rahmen der Reifenfortentwicklung vielfältig durchgeführt. Unterschiedliche Berechnungsmodelle werden angewendet. Beispiele hierfür sind die Aufsätze von Berger und Heinrich [Berger00] und Barquins [Barquins92]. Packmustergeneratoren und die dreidimensionale Erkennung von Packmustern sind Gegenstand von Forschungsarbeiten. 


\section{Anwendungen des Traction-Grippers}
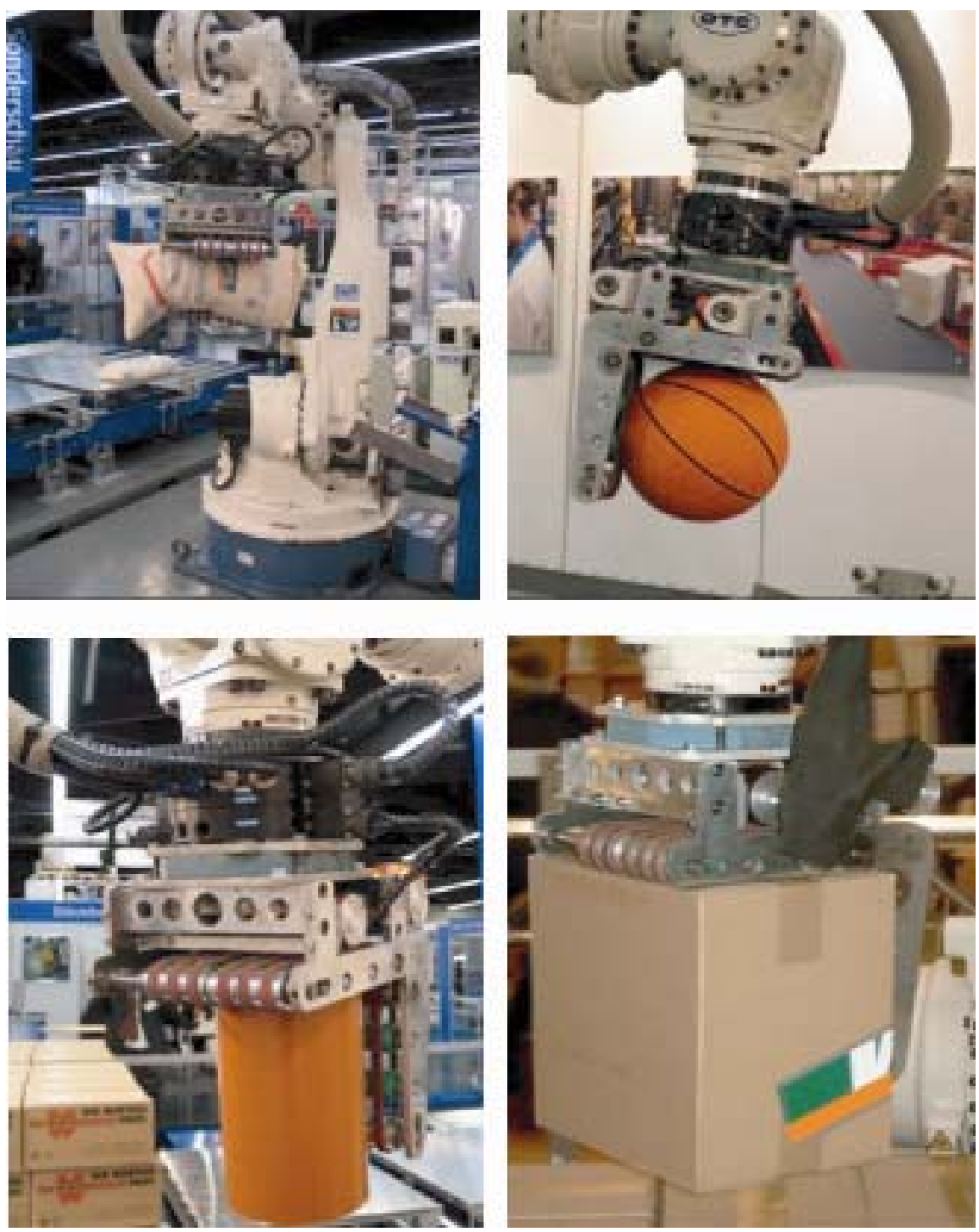

Abb. 29: Greifsystem Traction-Gripper im Einsatz mit Knickarmroboter beim Handling unterschiedlicher Objekte 


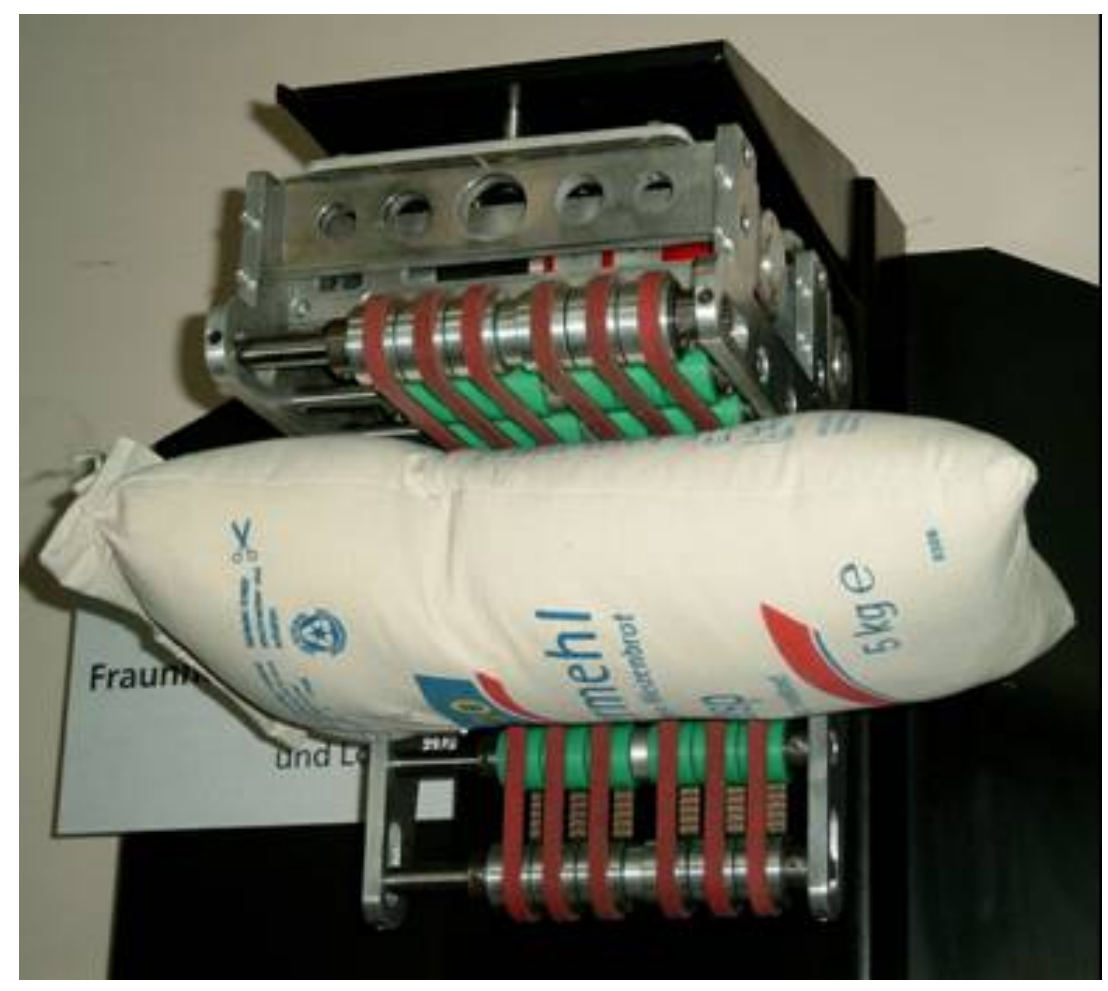

Abb. 30: Versuchsaufbau im Fraunhofer IML

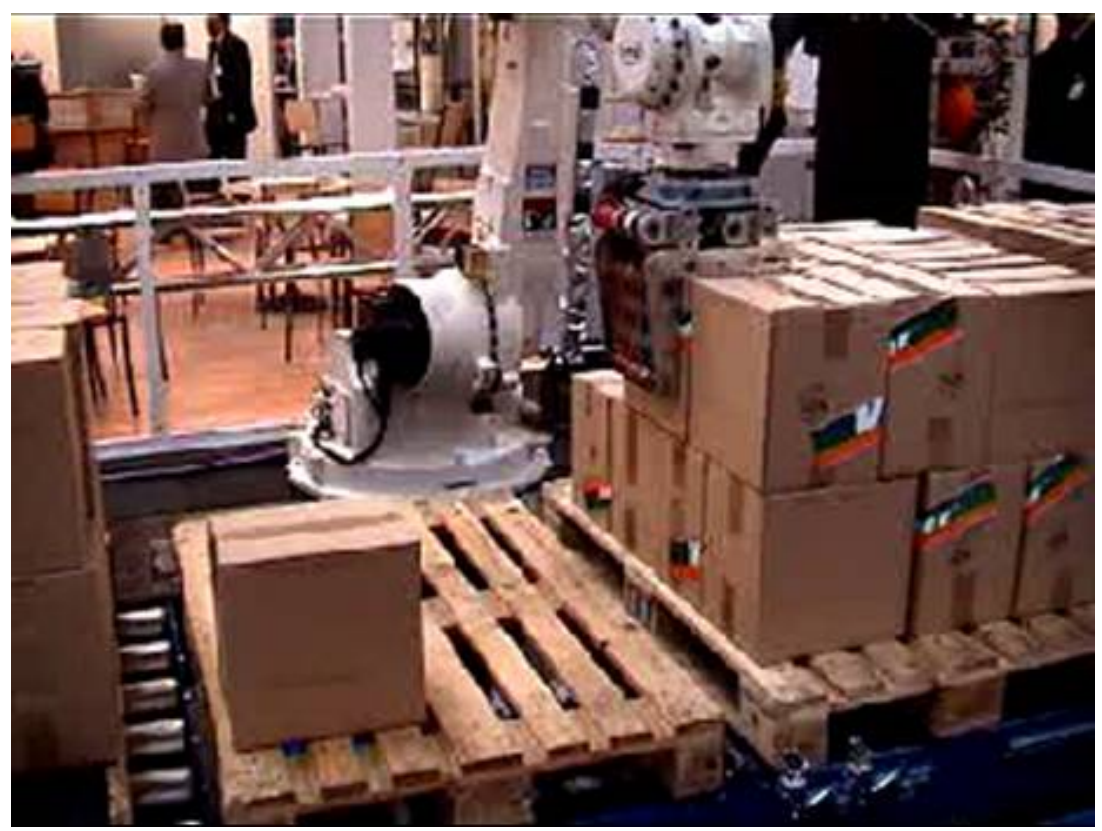

Abb. 31: Einsatz des Greifers beim Depalettieren, Hannover Messe 2005 


\section{Weiterführende Arbeiten}

Zur Weiterentwicklung des Greifsystems können folgende Arbeiten ausgeführt werden.

\section{Optimierung der Friktionsriemen}

Eine Maximierung des Reibwertes zu unterschiedlichen Stoffen als wichtigster Einfluss auf die Halteeigenschaften sollte nicht nur unter Berücksichtigung des Riemenwerkstoffes erfolgen. Einflüsse von Gleitgeschwindigkeit und Flächendruck sollen, entsprechend den Erkenntnissen der Gummireibung, Beachtung finden,. Eine Optimierung des Reibwertes darf nicht auf Kosten der Verschleißfestigkeit erfolgen.

\section{Verbesserungen durch artikelspezifische Einstellungen}

Durch artikelspezifische Einstellungen kann das Einsatzfeld des neuen Greifsystems erweitert werden. Eine automatische Einstellbarkeit der maximalen Riemenumfangskräfte ermöglicht Artikelwechsel ohne Umrüstzeiten. Bei Entnahme von Artikeln mit sehr unterschiedlichen Höhen aus Verbundstapeln ist eine Verstellbarkeit insbesondere der vertikalen Greiferbacke vorteilhaft. Auch diese Verstellung kann bei Bedarf automatisch realisiert werden. Einstellungen für die Backenlänge der horizontalen Greiferbacke und den Winkel zwischen Horizontal- und Vertikalgreiferbacke sind ebenso denkbar, vermutlich jedoch nur für Sonderfälle sinnvoll.

\section{Verbreitung des Systems}

Zur weiteren Verbreitung des Systems ist ein Piloteinsatz unter Produktionsbedingungen und Zusammenarbeit mit Roboter- und Peripherieherstellern geplant. Neben dem Entstapeln werden weitere potentielle Einsatzfälle gesucht.

\section{Literatur}

[Barquins92] Barquins, Michel: Adherence, friction and wear of rubber-like materials, In: Wear - An International Journal on the Science and Technology of Friction, Lubrication and Wear; Band 158 (1992) Heft 1-2, S. 87-117

[Berger00] Berger, H.R; Heinrich, G.: Friction effects in the contact area of sliding rubber... ; In: Kautschuk, Gummi, Kunststoffe; Band 53 (2000) Heft 4, S. 200-205

[Eberhardsteiner98] Eberhardsteiner, Josef; Fidi, Wolfgang; Liederer, Werner: Experimental determination of adhesive friction properties of rubber on plain surfaces; In: Kautschuk, Gummi, Kunststoffe; Band 51 (1998) Heft 11, S. 773-781

[Klüppel98] Klüppel, Manfred; Alshuth,Thomas; Schuster, Robert H.: Theorie der Gummireibung. (Fortschritt-Berichte VDI, Reihe 12: Verkehrstechnik/ Fahrzeugtechnik; Band 362) VDI-Verlag, 1998, S. 1-13

\section{Patente}

EP 0462518 A1 „Verfahren und Vorrichtung zum Aufnehmen bzw. Absetzen von paketförmigem Gut“"

EP 0627373 A1 „Verfahren und Vorrichtung zur Aufnahme von Stückgut“

DE 10338793.5 „Greifsystem für im Verbund gestapelte Objekte“ 